\title{
Paysager le texte colonial : de l'enclave historique à la relation esthétique
}

Putting Colonial Writing Back into Its Context: From the Historical Point of View to the Esthetic Vision

\section{Aurélie Dinh Van}

\section{OpenEdition}

Journals

Édition électronique

URL : http://journals.openedition.org/recherchestravaux/932

ISSN : 1969-6434

Éditeur

UGA Éditions/Université Grenoble Alpes

Édition imprimée

ISBN : 978-2-37747-014-3

ISSN : 0151-1874

Référence électronique

Aurélie Dinh Van, «Paysager le texte colonial : de l'enclave historique à la relation esthétique »,

Recherches \& Travaux [En ligne], 91 | 2017, mis en ligne le 01 octobre 2017, consulté le 08 septembre 2020. URL : http://journals.openedition.org/recherchestravaux/932

Ce document a été généré automatiquement le 8 septembre 2020

(c) Recherches \& Travaux 


\title{
Paysager le texte colonial : de l'enclave historique à la relation esthétique
}

\author{
Putting Colonial Writing Back into Its Context: From the Historical Point of View \\ to the Esthetic Vision
}

Aurélie Dinh Van

1 La relecture de l'œuvre de Saint John Perse par P. Chamoiseau pose la question de la réactivation d'un patrimoine, auparavant relégué dans une réception essentialiste : le texte persien, lorsqu'il est acculé au référent colonial, demeure rivé à un orgueil conquérant. La logique élitiste dans laquelle il est d'abord posté sert une cartographie hiérarchisante des frontières mentales: les territoires délimités obéissent à la dialectique d'un clivage dominant/dominé. Il s'agira ici de montrer que l'actualisation de l'œuvre persienne par l'un des tenants de la créolité dépend non seulement de l'expérience que celui-ci a du monde qui l'entoure, mais encore de la vision qu'il a de l'histoire. Le revirement d'opinion vis-à-vis d'un auteur, au départ rangé dans le tiroir impérialiste, est à la fois redevable d'une relecture du monde et d'une réévaluation de son rapport à l'altérité oppressante. Pour accepter de lire Perse, le lecteur antillais doit ajuster le regard qu'il porte, tant sur la dynamique de l'histoire, que sur les lieux de la colonisation, c'est-à-dire, dans le cas de Chamoiseau, sur les lieux qu'il habite encore et qui sont ceux de son enfance. Alors que la non-lecture de Perse est symptomatique d'une hantise de la violence coloniale et de la brutalité sourde de la départementalisation, l'accès au texte persien dépend au préalable de la mise en relation des imaginaires antagonistes, rendue possible par la médiation d'Édouard Glissant. La relecture de Perse dépend de cette idée-clé selon laquelle le divers de l'interprétation naît du conflit entre le conquérant glorieux et le subalterne déchu de son essence. C'est la relégation du texte persien au référent colonial qui va conditionner sa relecture créolisante, nécessairement corrélée, quant à elle, à la conception du monde comme un Tout-relié. 


\section{La mélancolie postcoloniale : une fabrique d'archétypes traumatiques}

2 Une logique de domination hante la première relation de Chamoiseau au texte persien. L'écrivain martiniquais range d'emblée ce texte du côté de la célébration de l'imaginaire colonial. L'épopée est ainsi envisagée dans une perspective de conquête de territoires vaincus. Elle prend délibérément part à l'élaboration du mythe fondateur de l'expansion outre-mer de l'Europe. Parallèlement, sa visée édificatrice sous-tend une généalogie racinaire qui préconise la déférence vis-à-vis de l'ascendance pionnière et offre le primat aux origines. L'antithèse archétypale entre la prétention à la pureté aristocratique chez celui qui se réclame d'un haut lignage et «l'impur esclavagiste ${ }^{1}$ » conditionne d'office une non-lecture récalcitrante :

Pour Perse, réaction de rejet immédiate de ma part : c'est un béké ! Il n'est pas des nôtres! (CPG, p. 13)

Cette réaction est symptomatique d'une " construction de soi en négatif ${ }^{2}$ », qui est aussi celle du Vieux guerrier dans Écrire en pays dominé : la lecture traumatique de l'histoire programme un a priori mélancolique qui s'inscrit en dissidence vis-à-vis de celui qui incarne l'autorité coloniale :

Des réflexes retournés contre soi-même, des recours rassurants au moule traditionnel qui embrigadent toute littérature et confèrent l'illusion de résister. Or, là on ne résiste pas: on s'arc-boute dans une ornière de soi tandis que la domination silencieuse se pare de modernité progressiste, d'ouverture démocratique et de vertus économiques imparables. Devant ce phénomène, je me trouvais désemparé. ( $C P G$, p. 21)

4 Les relents traumatiques de l'histoire dans la conscience subalterne se poursuivent dans l'appréhension amère de l'actualité dont le lecteur insurgé trouve qu'elle est régie par l'ascendant assimilationniste de la métropole sur les départements d'outre-mer : "La dépendance (l'idée même d'“outre-mer" et l'opposition inefficiente à elle) est devenue notre système d'existence dans l'ombre de la France » (CPG, p. 22). La rancœur subalterne vis-à-vis de l'épopée coloniale perdure ainsi dans une posture insurrectionnelle à l'encontre de la mainmise départementale, « frappe indolore » (CPG, p. 20) qui porte en germe « les lancinements de la domination brutale » (ibid.). Dans l'un et l'autre cas, l'accélération de l'histoire précipite un relent mélancolique qui ressasse l'épreuve de l'oppression selon un déterminisme fataliste.

5 La non-lecture de Perse manifeste un réflexe communautaire qui commande à l'œuvre sa catégorisation au sein d'un horizon d'attente culturel tributaire de l'ancrage dans l'histoire. Elle postule une continuité sournoise de la «mise sous relation» des Antilles: la domination brutale, celle de l'esclavage, se répète, se répercute dans le présent sous la forme de violences désormais lancinantes, qui sont celle de la départementalisation que le Vieux guerrier décrit comme "l'unique voie d'échappée aux plantations concentrationnaires » (EPD, p. 239) et celle du cyberespace que le même fustige comme l'appareil d'une « hypnose relationnelle » (EPD, p. 248) orchestrée par le centre diffus d'une «domination-qui-ne-se-voit-plus» (CPG, p. 22). Le ressentiment postcolonial en tant que mal-être identitaire, reproduit la compartimentation du monde soumis à la "frappe impérante ${ }^{3}$ " (EPD, p. 49) du colonialisme. Cette configuration territorialisante du monde délimite les nations et classifie les appartenances : les textes sont ainsi reclus au sein d'une territorialité façonnée par la 
tragédie de l'histoire. Au regard de cette représentation dialectique du monde, Perse est d'emblée posté dans l'archétype du béké orgueilleux, du conquérant altier. Il rejoint là l'impérialisme du Prince, il est « [l]'homme de l'universel conquérant, orgueilleux et hautain » (CPG, p. 15).

\section{Transmission du patrimoine : la dynamique destructrice d'un rapport complexe à un canon dépassé}

6 La question qui se pose alors est celle de comprendre comment la lecture actualisante de Perse tire profit de cette non-lecture mélancolique. Il s'agit pour ce faire de garder à l'esprit que l'œuvre de Perse comme entité impériale a compté dans sa réception, et que cette autorité qu'elle incarne au regard de l'histoire coloniale doit être resituée et actualisée dans une perspective contemporaine. En d'autres mots, comment faire lien avec un texte qui demeure apparemment incompatible avec la créolité de ses lecteurs ? Car il n'est pas question pour Chamoiseau de négliger le contexte de l'œuvre, de passer outre son impulsion scripturaire, qui lui fait prendre assise au sein d'un imaginaire colonial. L'autorité que l'œuvre incarne dans l'histoire n'est pas niée ni passée sous silence. Elle est au contraire reconnue, voire confortée.

7 La relecture de l'œuvre de Perse est ainsi sous-tendue par un imaginaire de la reviviscence et de la régénération, voué à disqualifier les relents territoriaux qui conditionnent son rejet :

La mort est dans la vie, la vie est dans la mort, l'une peut précéder l'autre, l'autre peut anticiper l'une, souvent leur mélange impensable avive quelque rémanente écaille d'une beauté. Et très souvent, pour les poètes, la mort fait émerger de l'œuvre d'inépuisables magnificences. (CPG, p. 12)

La métaphore germinative pose le contexte initial comme la condition de la réactualisation de l'œuvre assignée à une réception "par les racines». Ainsi le texte colonial est-il susceptible de "semer des graines, [d']offrir des graines, [de] prendre le bain qui désamarre des déveines secrètes" ( $C P G$, p. 11). Cette logique d'ensemencement, qui redonne vie au texte en dormance, offre un intérêt considérable dans la manière de réévaluer le processus de réactualisation de l'œuvre persienne. Pour cela, il ne s'agit pas de faire fi absolument de la lecture originelle, ce qui reviendrait à refuser son ancrage dans l'histoire : soustraire l'œuvre à son interprétation de départ équivaudrait à l'arracher de son contexte en la dénaturant. Mais il ne s'agit pas non plus de l'exclure d'emblée sous prétexte qu'elle est aux antipodes des revendications de ses non-lecteurs ou tout simplement de leur posture dans le monde. Cela reviendrait à la bannir tout à fait de sa mémoire, à la mettre au ban de sa bibliothèque sous prétexte qu'elle s'inscrirait en marge du vécu et du système de valeurs et de pensées de ses nonlecteurs, sans aucune possibilité de réhabilitation au regard de leur territoire mental. Par conséquent, l'actualisation de l'œuvre persienne n'écarte pas sa première lecture. Si elle présuppose la proclamation de la mort patrimoniale, c'est dans la perspective de mettre en jeu, après coup, le potentiel déflagratoire d'une œuvre au départ évincée. Pour relire Perse, il faut faire le constat de sa mort, dire l'inadéquation de sa réception première. Cela implique de rediriger le mythe aristocratique dans le sens d'une mythologie postcoloniale et de réévaluer ainsi le genre de l'épopée. Paysager le texte de Perse pose qu'au préalable le lecteur antillais soit en mesure de «capter la différence » 
(CPG, p. 36), de s'accaparer l'écart qui le tient à distance de lui. Cet écart s'ancre dans l'histoire coloniale, mais se répercute aussi dans la politique d'aujourd'hui : «Ici, en Martinique, rien ne nous dissimule la dépendance, sinon la dépendance elle-même car elle est en nous. » (CPG, p. 37) La non-lecture, qui fige la production-réception première dans l'enclave de l'histoire, constitue un point de rupture inaugurant le consentement à la relation.

\section{De l'universalisme conquérant à la poétique transfrontalière}

9 Le regard neuf porté sur l'actualité suscite la relecture d'un texte, au départ, rivé à une posture orgueilleuse face au monde. C'est en reconsidérant le monde dans lequel il vit que le lecteur se donne les moyens de faire revivre un texte au départ raidi par son appartenance à une communauté d'assignation. Par l'entremise de Glissant, Chamoiseau découvre en Perse un Poète dont l'élévation altière aspire à une créolisation du monde. «Partout, le règne d'une oppression indépassable » (CPG, p. 24) : si Chamoiseau fait le constat d'une «mise sous relation » du monde, c'est pour mieux montrer que le seul exutoire à l'enclave de l'Histoire ne peut reposer que dans l'exaltation d'une grandeur épique qui assujettit le monde à son centre. L'assaut des éléments, ce que le poète de Fort-de-France nomme "l'intempérie omniphone » pour désigner « l'élévation, les vents, le tournoiement, l'éclair » (ibid.), va constituer la clé de voûte pour la relecture de l'épopée persienne. C'est par ce biais-là que Chamoiseau parvient à reconsidérer les lieux, l'histoire et les assises idéologiques qui sous-tendent le texte de Perse. Cette remise en question de l'univocité épique conditionne une relecture topographique qui aura pour but de conjurer les traumatismes coloniaux. Il ne s'agit pas de décoloniser le patrimoine, mais bien plutôt de tirer parti de sa raideur canonique. De quelle manière? En paysageant sa fulgurance, son incandescence, en offrant une lisibilité densifiée à l'expansion coloniale, vue non plus comme un tracé droit, monolingue, mais comme un écosystème relationnel.

Dans cette perspective postcoloniale, le culte généalogique de Perse, célébrant l'ascendance aristocratique et confinant à la pureté de l'origine, n'est plus envisagé dans une stricte logique racinaire. Paradoxalement chez lui -c'est ainsi que Chamoiseau le comprend - la tragédie de l'histoire hantée par l'inflation de l'impur, par l'avilissement de la condition humaine, trouve à se désamorcer dans l'éloge de la caste et de la suprématie initiale. La déliquescence de l'homme due à l'abomination esclavagiste va désormais trouver son échappatoire dans la surenchère élitiste d'un haut lignage :

Cette obsession de la pureté dans Exil chez Perse (confirmée par l'élévation de toute son œuvre dans une désincarnation orgueilleuse) permet de supposer que l'instant de l'amorce, l'excoriation inaugurale, le lieu de l'inflammation, se trouve certainement dans l'impur : le décrépi de son habitation de naissance, la perception d'une trouble proximité avec ces nègres régresses hindous (grandes bêtes taciturnes qui habitent le domaine et s'ennoblissent ainsi), la damnation esclavagiste, racismes et bâtardises, comme fondation de ce nouvel espace... Fuir le trouble des créolisations, l'impur esclavagiste... Creusement encore, inflammation toujours: matrice sévère pour tous. ( $C P G$, p. 21$)$ 
11 L'épopée comme mythe fondateur de la suprématie européenne n'a plus valeur de conquête. Ici s'opère un revirement axiologique du pur et de l'impur. La recherche de la pureté ne vise désormais plus qu'à saper et à déjouer « l'impur esclavagiste » (ibid.) :

Parfois, il m'arrive même de penser que le recueil Éloges, où le poète Perse explore son enfance antillaise, avec louanges, célébrations, fêtes, émerveilles, et estime constante, serait en fait une sorte de purification poétique du Lieu. Comme une conjuration de ce qu'il va s'efforcer de tenir à distance durant toute sa vie, toute son œuvre, à commencer par la Guadeloupe. (Ibid.)

L'épopée cathartique reconfigure les lieux de l'oppression, ceux de l'histoire, qui sont aussi ceux qui désignent l'expérience vécue par le lecteur antillais. Ce qui est en jeu, c'est le rapport que celui-ci a avec le territoire insulaire qu'il habite et qu'il doit à présent départir de son traumatisme colonial. L'éloge du lieu insulaire chez Perse va ainsi permettre à Chamoiseau d'exorciser les attaches biographiques et historiques qui le lient à son île. L'auteur doit renoncer à la permanence du lieu de son enfance et déborder la fatalité insulaire de l'enfermement. Pour redonner vie au huis clos propre au monde des plantations, il doit conjurer la déperdition de l'île consacrée comme pays :

Me promener dans Fort-de-France, ma ville. Voir et revoir les lieux d'enfance vieillis, usés, qui m'attestent que des usures similaires se sont produites en moi, même si je ne les vois pas et que mon esprit s'illusionne encore d'une permanence. Vieillir en lieu d'enfance c'est s'exposer à l'intangible de l'esprit, chimère agile parmi les ruines qui s'étendent, l'illusion de l'écume fraîche dans les songeries vasardes du marigot. (CPG, p. 22)

Le vieillissement de Fort-de-France met au jour le simulacre d'espoir corrélé au lieu d'enfance. L'île claustrée, prise dans l'étau de l'accélération de l'histoire, doit s'affranchir du poids de son sépulcre. À charge pour le lecteur antillais, de saper les limites mortifères d'une géographie de la prédation. À l'ankylose de la conscience sous le fardeau de l'histoire, succède le parti pris d'un état de veille émancipatoire qui le tire vers un « [a]ller libre» (CPG, p. 14) :

Je ne suis pas le seul à être éveillé-réveillé dans ce sommeil de pays dominé, ni même le plus lucide d'entre nous. Mais j'ai l'impression d'accueillir l'onde des veilles silencieuses, diffuses au travers des grandes mornes. Je suis réceptacle. Je suis capteur. Aboutissement de l'influx d'un mal-être. Petit point d'eau dans les déserts de cette blessure que Césaire désignait comme jamais refermée. (Ibid.)

Car ces lieux s'ancrent au préalable au sein d'un dispositif cynégétique, qui accule l'esclave fugitif au gouffre marin. L'île est d'abord, dans l'imaginaire antillais, un huisclos mortifère. On pense par exemple à l'aporie du marronnage chez Glissant, lequel présente l'esclave marron butant contre une insularité close ${ }^{4}$. La dimension catastrophique de la fuite en fait une course tragique que précipite une traque implacable. De même, chez Césaire, le paysage insulaire est hanté par la meurtrissure coloniale :

Je savais à quel point, pour Césaire, le paysage supporte la marque de la plaie coloniale, et combien l'île décrite ainsi signifiait en grande part l'étau d'une domination raide. (EPD, p. 261)

15 Mais l'épopée du planteur déplace le point de vue de l'opprimé et lui offre la voie d'une échappatoire. L'̂̂le natale, piège tragique, est transmuée par Perse en «marchepied d'envol » (CPG, p. 263), comme si la liberté de Perse augmentait la mobilité créole de son lecteur, comme si elle défaisait les traumatismes de la plantation et de la traversée. De la même manière, la mer, «masse geôlière » (EPD, p. 262), lieu d'avalement hanté 
par la disparition et l'absence, est «[e]n Perse, [...] élément de puissance, source de connaissance, présomption de l'esprit... » (EPD, p. 263), aire de trafic et de transaction marchande. C'est là que le système des valeurs coloniales dont Perse se fait le chantre est inversé : la lecture de Perse augmente la catharsis du lieu insulaire et du gouffre atlantique. L'exiguïté tragique de la cale négrière et de la plantation esclavagiste est entravée par la vigueur épique, de sorte que Glissant a pu dire que ce revirement axiologique s'est s'opéré à l'insu de son auteur. Si l'œuvre de Perse incarne à première vue la suprématie de l'ordre colonial en valorisant la trajectoire conquérante des découvreurs, elle n'en porte pas moins en germe une vigueur relationnelle qui paysage le monde conquis en interface cosmogonique :

Et pourquoi partagé, sinon qu'il a, malgré son dire, greffé en lui le fourmillement, le bienheureux fertile (l'opulent, le grouillement) de ce qui à nouveau naît ? Partagés, son appel d'horizon, ses espaces démesurés, ses ascensions de terres en terres. Partagé, l'impact du monde ${ }^{5}$.

Perse paraît destiner l'« Occident immobile ${ }^{6}$ » à satisfaire un projet universaliste, mis au service des valeurs d'un centre atavique. Mais c'est compter sans le fait que ces convictions coloniales célébrant une vision européo-centrée du monde portent la trace de ce que Chamoiseau nomme un « respir poétique du monde » (EPD, p. 263):

Immobile, pour cet appétit des œuvres stables qui hante les Aventuriers. Immobile parce qu'il [Perse] a le souci des valeurs qui peut-être se défont sourdement de lui, et dont il psalmodie avec une solennelle rigueur la permanence et la pérennité ${ }^{7}$.

On voit que le fatalisme territorial, par l'impulsion épique de Perse, s'ouvre désormais à un écheveau d'imaginaires féconds, de même que "le tapis sous-marin de cadavres " (EPD, p. 264) se transmue en "aire de relations» (EPD, p. 265) et "miroite d'une mosaïque d'imaginaires» (ibid.). La densification des communautés assujetties tient paradoxalement son descellement de l'étau colonial : «Ces diversités, projetées de tous les continents, vont se rencontrer dans le cadre le plus clos qui soit: la plantation esclavagiste. » (CPG, p. 19) Quant à la traversée, on retrouve à son sujet cette diffraction des imaginaires emprisonnés : « Cette diversité initiale (en termes de dieux, de langues, de traditions) s'est retrouvée concassée dans une bouillie humaine au fond de la cale négrière. » (Ibid.) Le point de vue géographique de Perse diffère ainsi de l'imaginaire antillais, car c'est celui d'un homme qui est bel et bien délivré des amarres biographiques le vouant au pays natal. Les lieux de l'histoire sont réévalués au prisme de l'émancipation ontologique: Perse est "soucieux d'effacer les rives, les berges, les centres et les périphéries qui auraient pu le désigner de quelque part » (CPG, p. 22). Il s'invente un mythe personnel qui vise une reconfiguration postcoloniale du monde. L'échappée insulaire, qui au départ est mise au service d'une quête de l'origine, soustend la prophétie d'un cosmopolitisme cosmique. Si Perse est "homme d'Atlantique " (ibid.), c'est dans une double perspective contradictoire, élitiste d'abord, qui conforte la suprématie européenne, cosmique ensuite, qui situe la traversée dans une aire de " promulgation divine » (EPD, p. 263):

Homme d'Atlantique, se dira-t-il, pour mieux s'éloigner des bâtardises créoles et dissiper toute idée de tanière. Homme d'Atlantique aussi car c'était son Lieu de relation aux grandes forces du monde. (CPG, p. 48)

18 De même, l'appropriation inaugurale de l'île dans Éloges ne vise pas la célébration du mythe de l'enfance dans l'éden tropical, elle donne plutôt son impulsion à une épopée cosmogonique affranchie des lieux : 
Ainsi, son insularité natale inaugure son œuvre (Images à Crusoé, Éloges) avant d'en disparaître. Il se met, comme il l'a dit lui-même, à habiter son nom, pour mieux quêter son universalité désincarnée qui inventorie des fastes inépuisables. (EPD, p. 263)

19 Il y a, à l'insu de l'atavisme colonial de Perse, la quête d'un débordement des « fatalités de la terre natale » (CPG, p. 74). Perse exorcise la terre en deuil en inventant un « Lieu presque hors sol: Francité, Occident, Atlantique»(ibid.). L'«obsession de la pureté » déjoue à présent la quête ontologique. L'orgueil aristocratique qui sous-tend l'édification du mythe personnel oblige à une traversée sans attaches, qui fait du cosmos l'intercesseur d'une conscience rendue désormais disponible au monde :

Le relisant de plus près, je m'aperçus que Saint-John Perse dispose, en terre créole, d'une liberté fondamentale. Du fait d'une position dominante de béké, il n'a rien à prouver à dénoncer à refuser comme le nègre Césaire - nié dans son humanité doit le faire. [...] C'est pourquoi, face à la mer chantée de manière continuelle, Perse aura vision, non du mur d'isolement de l'imagerie occidentale, mais d'une vaste perspective vers une conquête du monde, ou plutôt: d'une aire frémissante vers l'universalité altière qui commande à son souffle. (EPD, p. 262)

L'épopée cosmogonique transforme l'ordonnancement colonial du monde en terre désormais habitable qui facilite les «magnétismes entre les différences » (CPG, p. 99). L'écheveau créolisant que Chamoiseau désigne comme un processus d'«alchimie anthropologique» (CPG, p. 20) tire son origine de l'inventaire mélancolique des aliénations et des résistances : "Perse rejoint le monde par l'élévation universaliste. » (CPG, p. 159) Ce descendant de pionniers se change ainsi en guerrier de l'imaginaire. L'élan impérial vise la déflagration du lieu de l'histoire en un nouvel «écosystème mental » (CPG, p. 28) qui tient compte du charroi des narrations. Alors que l'entreprise de la décolonisation implique de dénaturer le texte en le déliant de sa dimension impériale, l'actualisation paysagère pose l'incompatibilité radicale entre des altérités foncièrement antagonistes comme le catalyseur du divers. Décoloniser le texte de l'altérité dominante, c'est chercher à tout prix à renverser sa force impériale en la retranchant dans l'oubli. Cette opération inflexible implique un vis-à-vis au cours duquel le lecteur "mélancolique » s'efforce absolument de saper le contexte et d'en abolir la négativité, comme si exclure le livre de sa bibliothèque permettait de désamorcer la charge traumatique de l'histoire. L'actualisation, au contraire, ne nie pas le contexte. Elle prend acte d'un écart irrémédiable qui sépare le lecteur antillais du texte colonial et en tire profit. En d'autres termes, l'actualisation s'appuie sur l'ampleur de l'épopée qu'elle ne réduit pas à un mythe des origines, mais dont elle se sert pour affirmer sa vigueur transfrontalière. Le point de vue altier, celui de Perse béké, non seulement déplace le regard du colonisé, mais encore reconfigure les lieux clos de sa mémoire. La transmission d'un canon passé sous-tend ainsi une logique de déconstruction qui permet de réévaluer l'empreinte que celui-ci laisse dans les imaginaires sous tutelle. L'épopée du conquérant nourrit la mise en récit postcoloniale du monde et augmente la conscience d'une créolité qui resolidarise les altérités incompatibles. Pour Chamoiseau, l'écart qui sépare le lecteur antillais du texte colonial est du même ordre tactique que celui que vit l'esclave des plantations. On dira en effet que cet aliéné vaincu ne parvient à marronner et à ruser que dans un écart dramatisé, où la promiscuité insoutenable (celle du lieu plantationnaire) le tient à une distance « imprévisible », pourrait-on dire, du maître. Actualiser le texte colonial, c'est donc aussi réévaluer le territoire forclos de l'île, dont la mesure tragique facilite la mise en contact des imaginaires antagonistes. C'est aussi conjurer la béance atlantique qui place 
la traversée sous le régime de la catastrophe. La relecture de Perse désamorce le carcan des lieux, de sorte que la mer abyssale et la terre confrontée au gouffre sont désormais les lieux d'un territoire mental en transaction. Celui-ci accède, émerveillé, à son propre paysage.

\section{NOTES}

1. P. Chamoiseau, Césaire, Perse, Glissant. Les liaisons magnétiques, Paris, Philippe Rey, 2013, p. 21. Ensuite abrégé $C P G$.

2. P. Chamoiseau, Écrire en pays dominé, Paris, Gallimard, coll. «Folio», 1997, p. 249. Ensuite abrégé EPD.

3. La « frappe impérante » désigne la violence ordonnatrice d'une régie univoque du monde, celle que le colonialisme a infligée aux territoires qu'il a conquis et administrés.

4. Voir EPD, p. 264 : «Chez Glissant, une scène perdure : celle du Nègre marron qui, au bout de sa fuite, bute sur l'infranchissable inconnu de la mer. Pour les esclaves traqués par des dogues et des milices armées, la mer devait représenter ce que signifiait la forêt aux yeux des bagnards de Guyanes : une masse d'enceinte vivante qui avalait ses proies. Une verticale sans perspective et sans promesse de libertés.

5. É. Glissant, L'Intention poétique, Paris, Gallimard, 1997, p. 108.

6. Ibid.

7. L'Intention poétique, ouvr. cité, p. 108.

\section{RÉSUMÉS}

Lorsque P. Chamoiseau propose de relire l'œuvre de Saint-John Perse, c'est bien dans une perspective postcoloniale dont il faut mesurer les enjeux. L'actualisation du texte colonial invite non seulement à revisiter les lieux de l'enfance (communs au lecteur antillais et à l'auteur béké), mais encore à réévaluer l'enclave de l'histoire (coloniale qui se reproduit dans l'actuelle mainmise départementale). Glissant joue à cet égard le rôle d'intercesseur, puisqu'il envisage la mobilité créole du Tout-monde comme un processus de densification des identités. Ainsi, c'est en reconsidérant la mémoire traumatique dont il a hérité et en paysageant le monde dans lequel il vit, que le lecteur antillais peut parvenir à faire revivre un texte mort et relégué dans un tiroir d'orgueil.

This article examines the postcolonial perspective, which P. Chamoiseau adopts when referring to the works of Saint-John Perse. In order to reconfigure Perse's colonial texts, Chamoiseau is not only invited to revisit the places of his childhood (common to both authors), he also needs to reassess the enclave of history (since colonial history repeats itself as the Departement status currently allows to maintain a strong grip on the Caribbean people). Glissant, who conceives of a 
Creole mobility of "Tout-monde" as a densification of identities, acts in this respect as an intercessor. Indeed, by reconsidering traumatic memory he has inherited and by landscaping the world he lives in, the Creole reader is able to revive a text that appears at first to be dead and solidified by vanity.

\section{AUTEUR}

\section{AURÉLIE DINH VAN}

Aurélie Dinh Van est professeure certifiée de lettres modernes, membre permanente du CNEP (Université de Nouméa) et membre associée du laboratoire LLA-Créatis (Université de ToulouseJean Jaurès). Elle a soutenu en 2014 une thèse intitulée Pour une écologie-monde de la littérature : relation esthétique et diversité culturelle, sous la direction de Gérard Langlade et Catherine Mazauric. Son travail de recherche porte sur les questions postcoloniales, les dynamiques transculturelles et la lecture littéraire. 